\section{Essential and separable roles for Syndecan-3 and Syndecan-4 in skeletal muscle development and regeneration}

\author{
D.D.W. Cornelison, ${ }^{1}$ Sarah A. Wilcox-Adelman, ${ }^{2}$ \\ Paul F. Goetinck, ${ }^{2}$ Heikki Rauvala, ${ }^{3}$ \\ Alan C. Rapraeger, ${ }^{4}$ and Bradley B. Olwin ${ }^{1,5}$ \\ ${ }^{1}$ Department of Molecular, Cellular, and Developmental \\ Biology, University of Colorado at Boulder, Boulder, Colorado \\ 80309, USA; ${ }^{2}$ Cutaneous Biology Research Center, \\ Massachusetts General Hospital, Harvard Medical School, \\ Charlestown, Massachusetts 02129, USA; ${ }^{3}$ Neuroscience \\ Center, Department of Biosciences, and Institute of \\ Biotechnology, University of Helsinki, Helsinki FIN-00014, \\ Finland; ${ }^{4}$ Department of Pathology and Laboratory Medicine, \\ University of Wisconsin-Madison, Madison, Wisconsin 53706, \\ USA
}

Syndecan-3 and syndecan-4 function as coreceptors for tyrosine kinases and in cell adhesion. Syndecan- $3^{-/-}$ mice exhibit a novel form of muscular dystrophy characterized by impaired locomotion, fibrosis, and hyperplasia of myonuclei and satellite cells. Explanted syndecan$3^{-/-}$satellite cells mislocalize MyoD, differentiate aberrantly, and exhibit a general increase in overall tyrosine phosphorylation. Following induced regeneration, the hyperplastic phenotype is recapitulated. While there are fewer apparent defects in syndecan-4 $4^{-/}$muscle, explanted satellite cells are deficient in activation, proliferation, MyoD expression, myotube fusion, and differentiation. Further, syndecan- $4^{-/-}$satellite cells fail to reconstitute damaged muscle, suggesting a unique requirement for syndecan-4 in satellite cell function.

Supplemental material is available at http://www.genesdev.org.

Received April 23, 2004; revised version accepted July 15, 2004.

Skeletal muscle in most vertebrates is a terminally differentiated tissue; thus, existing myonuclei are permanently postmitotic and are unable to participate in postdevelopmental growth or repair. When muscle is injured or diseased, damaged muscle must be repaired or completely regenerated. Satellite cells, so named because of their location beneath the basal laminae of differentiated myofibers, are a reserve myoblast population distributed throughout differentiated adult muscle; their embryonic origin and relationship to developmentally differentiating myoblasts remain unclear. Mitotically and physiologically quiescent in healthy muscle, they are stimulated by local damage to proliferate extensively and form a pool of replacement myoblasts that will subsequently

[Keywords: Muscle regeneration; satellite cell; stem cell; myogenesis] ${ }^{5}$ Corresponding author.

E-MAIL bradley.olwin@colorado.edu, FAX (303) 492-1587.

Article and publication are at http://www.genesdev.org/cgi/doi/10.1101/ gad.1214204 differentiate to form new muscle tissue (for reviews, see Schultz and McCormick 1994; Hawke and Garry 2001). The molecular mechanisms controlling this process are not understood, particularly the signaling pathways operating early in regeneration that lead to the initial activation and proliferation of the satellite cells and later to their induction of the myogenic program.

The receptor tyrosine kinase c-met and/or fibroblast growth factor (FGF)-dependent receptor tyrosine kinases are currently the best candidates for transducing these early satellite cell signaling events (Allen et al. 1995; Cornelison and Wold 1997; Tatsumi et al. 1998). Both receptors are absolutely dependent on heparan sulfate to form a high affinity ternary complex capable of transducing intracellular signaling events (Carey 1997; Ornitz 2000; Rapraeger 2000) and thus, it is significant that quiescent satellite cells also express the transmembrane heparin sulfate proteoglycans (HSPGs) syndecan-3 and syndecan-4 (Cornelison et al. 2001). There are four related members of the syndecan family, which are highly similar at the protein level through the transmembrane and intracellular domains but divergent in their extracellular domains (for review, see Zimmermann and David 1999). Although skeletal muscle cell lines express all four syndecan family members (Larrain et al. 1997; Olguin and Brandan 2001), developing myoblasts in the embryo express syndecan-1, syndecan-3, and syndecan-4 (Cornelison et al. 2001; Olguin and Brandan 2001), and adult satellite cells express only syndecan-3 and syndecan-4 (Cornelison et al. 2001). Previously, we have shown that disruption of heparan sulfate in cultured primary satellite cells results in delayed activation and delayed initiation of myogenesis (Cornelison et al. 2001). However, this effect could not be definitively attributed to the inactivation of any specific HSPG. Therefore, we examined the skeletal muscle and satellite cell behavior from mice carrying homozygous deletions of either syndecan-3 or syndecan-4. Unexpectedly, we found skeletal muscle and satellite cell defects that are unique to the loss of each syndecan, suggesting that they function to perform distinct yet essential roles in both skeletal muscle development and skeletal muscle regeneration.

\section{Results and Discussion}

Syndecan-3-/- animals have been shown to display feeding behavior (Reizes et al. 2001) and learning and memory abnormalities (Kaksonen et al. 2002); additionally, their hindlimb locomotion is impaired in our colony. Adult muscle in wild-type mice is characterized by uniformly packed myofibers of consistent caliber, a smooth and regular basal lamina, and the presence of sublaminar quiescent satellite cells identified by c-met staining (Fig. 1A-C). In contrast, syndecan-3-3 ${ }^{-1}$ adult muscle possesses extensive fatty infiltrates (fibrosis) both between and within muscle fibers (Fig. 1D). This phenotype resembles that common in other dystrophic muscle (Watchko et al. 2002). Innervation, as determined by the presence of neurofilament-labeled peripheral nerve bundles (Fig. 1E) and $\alpha$-bungarotoxin labeling of motor endplates (Supplementary Fig. 1), is qualitatively and quantitatively similar to wild type (Fig. 1B; Supplementary Fig. 1). Sublaminar (quiescent) satellite cells 


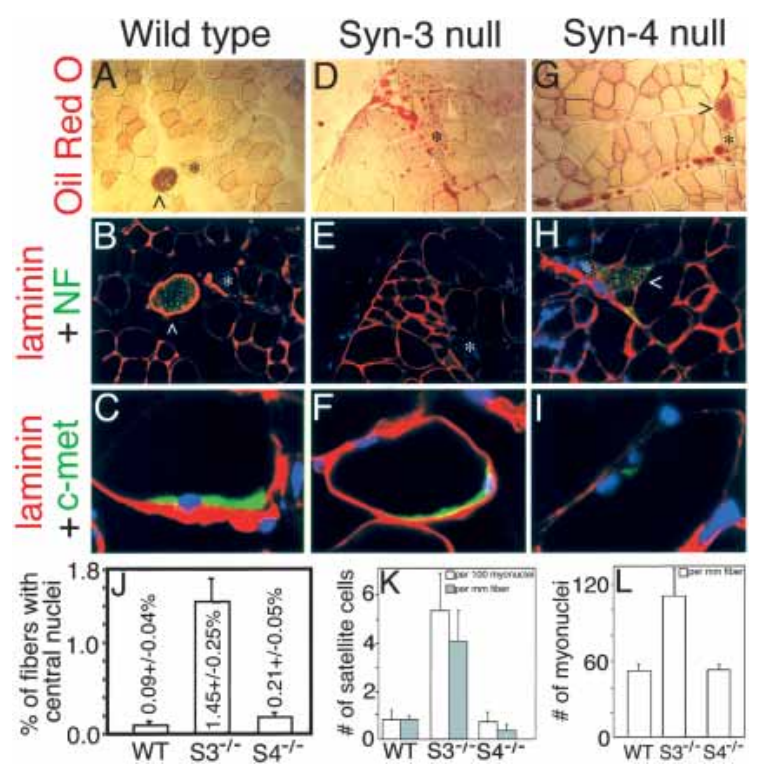

Figure 1. Morphological features of adult hindlimb muscle. Hindlimb muscle of adult wild-type $(A-C)$, syndecan-3-/- $(D-F)$, or syndecan-4 $4^{--}(G-I)$ mice was stained with the lipid marker Oil Red O $(A, D, G)$, with antibodies to laminin (red) and either the peripheral nerve protein neurofilament (NF; green; $B, E, H)$ or the satellite cell marker c-met (green; $C, F, I$ ); nuclei were visualized with DAPI. Asterisks identify blood vessels and carats identify peripheral nerve bundles. $(C, F, I)$ C-met-positive sublaminar satellite cells are present in all genotypes. Laminin in syndecan-4-/- myofibers appears both weaker and more diffuse than in either wild type or syndecan- $3^{-1-}$; compare $C$ and $F$ with $I$. (J) Quantification of the average percentage of myofibers containing central nuclei in sections through the quadriceps muscle indicates that syndecan- $3^{-/-}$muscles have undergone recent regeneration. $(K)$ When fiber-associated satellite cells were counted per 100 myonuclei and per millimeter of host myofiber,

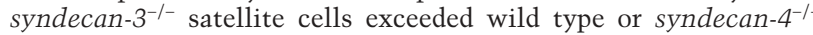
cells by five- to sevenfold. $(L)$ A twofold increase in myonuclear concentration was also seen in syndecan-3-1- myofibers over syndecan $-4^{-/}$and wild type. Bars in $J-L$ represent one standard deviation.

(Fig. 1F) appear morphologically similar to wild type from their size, position, and marker gene expression. A striking difference from wild type is the $>15$-fold increase in the number of regenerating myofibers indicated by centrally located myonuclei (Fig. 1J) in the syndecan-3 $3^{-/-}$muscle, suggesting that regeneration is ongoing in the absence of experimental damage. This is consistent with the locomotion defects we observe in syndecan $-3^{-/-}$mice and suggests that deletion of syndecan-3 may be implicated in a novel form of muscular dystrophy.

In contrast, syndecan-4-/- animals, which display a delay in epidermal wound healing and angiogenesis in granulation tissue (Echtermeyer et al. 2001) and defects in fibroblast adhesion and migration (Wilcox-Adelman et al. 2002), have a normal gait and do not display signs of chronic muscle regeneration. Examination of the tissue shows adipose infiltrates between but not within muscle fibers (Fig. 1G), as well as the presence of normal peripheral nerve bundles (Fig. $1 \mathrm{H}$ ) and normal motor endplate morphology (Supplementary Fig. 1). Although sublaminar satellite cells are present, they apparently display reduced levels of c-met (Fig. 1I) and syndecan-3 staining (data not shown). We also observe a reduction in thickness as well as an apparent disorganization of the myo- fiber basal laminae (Fig. 1, cf. C,F and I). Additionally,

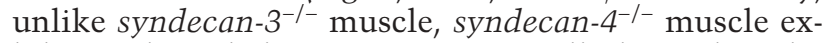
hibits only a slight increase in centrally located nuclei (Fig. 1J), suggesting that regeneration is not ongoing. Thus, our initial phenotypic characterization suggests that syndecan-3 and syndecan-4, surprisingly, appear to perform quite distinct functions in skeletal muscle development.

When single fibers from syndecan-3-/- muscle are examined, both differentiated myonuclei and satellite cell nuclei are present in great excess over wild-type counts (Fig. 1K,L), although neither fiber number per muscle nor average fiber diameter changes significantly (data not shown). When satellite cells (c-met-positive, myofiberassociated cells) and DAPI-stained myofiber nuclei are counted at $24 \mathrm{~h}$ postharvest (prior to the first satellite cell division) and the number of satellite cells per 100 myofiber nuclei is determined, a 6.7-fold increase over wild type is observed (Fig. 1K). When satellite cells and myonuclei are counted per millimeter myofiber length to control for the increase in myonuclear number, syndecan-3 $3^{-/}$satellite cells exceed wild-type cells by greater than fivefold (Fig. $1 \mathrm{~K}$ ) and syndecan-3-/- myonuclei exceed wild-type by at least twofold (Fig. 1L). This increase in both myonuclear and satellite cell numbers is particularly intriguing because it may indicate a possible inhibitory role for syndecan-3 signaling in myogenic precursors contributing to both the differentiated muscle and satellite cell. In contrast, the number of syndecan$4^{-/-}$satellite cells (Fig. $1 \mathrm{~K}$ ) and myonuclei (Fig. $1 \mathrm{~L}$ ) is indistinguishable from wild type, suggesting that such a putative role in developmental control of cell number is unique to syndecan-3. To determine if loss of one satellite cell-specific syndecan affected expression of the other syndecan, we examined expression of each syndecan in the opposite null phenotype. Expression of syndecan-4 in syndecan-3 $3^{-/}$fiber-associated satellite cells was comparable to syndecan-4 expression in wild type (Supplementary Fig. 2), whereas syndecan-3 expression in syndecan-4 $4^{-1-}$ satellite cells was, as seen in section stains, slightly reduced compared with wild type (Supplementary Fig. 2).

When examined in myofiber culture, wild-type and syndecan- $3^{-/-}$satellite cells are activated by $24 \mathrm{~h}$ postharvest, on the basis of emergence from beneath the basal lamina and expansion of their cytoplasm (Fig. 2A,D). Wild-type (Fig. 2B,C) and syndecan-3-null cells (Fig. 2E,F) both proliferate and express myogenic regulatory factors with normal timing. However, quantitative analysis of the satellite cells depicted in Figure 2 reveals that significantly fewer syndecan-3-/- satellite cells express MyoD than do equivalent wild-type cells (Fig. 3). The reduction in MyoD protein is frequently accompanied by mislocalization of MyoD to the cytoplasm of the satellite cell (Fig. 2, cf. C and F) that appears to be exacerbated over time (Fig. 3). Both the cause and the potential effects of this mislocalization of a transcription factor essential for appropriate satellite cell myogenesis (Cornelison et al. 2000) are unknown at this point.

When explanted into single fiber culture, syndecan$4^{-/-}$satellite cells initially appeared incapable of either activation or subsequent proliferation, with the majority of cells failing to expand their cytoplasm and to appear on top of the basal lamina, to begin MyoD expression by $24 \mathrm{~h}$ (Fig. 2G), or to occur in doublets at $48 \mathrm{~h}$ (Fig. $2 \mathrm{H}$ ) as both wild-type (Fig. 2A,B) and syndecan-3-/- (Fig. 2D,E) 

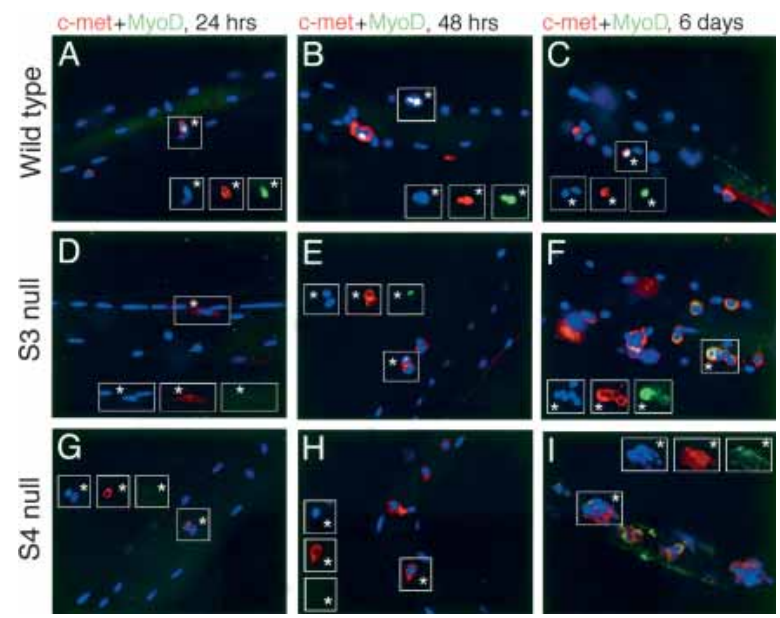

Figure 2. Expression of c-met and $\mathrm{MyoD}$ in fiber-associated satellite cells. After $24 \mathrm{~h}(A, D, G), 48 \mathrm{~h}(B, E, H)$, or $144 \mathrm{~h}(C, F, I)$ in culture after harvest, myofibers were fixed and stained for the satellite cell marker c-met (red) and the myogenic regulatory transcription factor MyoD (green); nuclei were visualized with DAPI. $(C, D, G)$ Note that some DAPI-stained nuclei in insets are myonuclei of the parent fiber and not satellite cells, and thus are not positive for any other stains. Wild-type satellite cells $(A-C)$ are activated by fiber harvest and can be distinguished from the host myofiber at $24 \mathrm{~h}$ by their morphology and expression of MyoD in some cells $(A)$. They divide synchronously between 24 and $48 \mathrm{~h}$ while the percentage of cells expressing MyoD increases $(B)$ and continue to proliferate on the host myofiber $(C)$ as well as in adherent colonies on the culture plate (see Fig. 5). $(D-F)$ Syndecan-3-1- cells displayed grossly normal phenotypes except that MyoD expression is somewhat reduced and is often mislocalized: Inset in $E$ shows two sister satellite cells, one that does not express MyoD and one with appropriate nuclear MyoD staining, whereas all of the satellite cells in $F$ display inappropriate cytoplasmic MyoD staining. (G-I) Syndecan-4 ${ }^{-/-}$cells displayed defective patterns of activation $(G)$, proliferation $(H)$, differentiation, and association $(I)$, as well as a low incidence of MyoD expression (cells in $G, H$ do not express MyoD), coupled with a high level of MyoD mislocalization (none of the clustered cells in I display nuclear MyoD staining; see Fig. 4). Asterisks label satellite cells.

cells do. However, by $6 \mathrm{~d}$ in culture, the syndecan- $4^{-/-}$ satellite cells have proliferated extensively on the fiber and most appear in large aberrant clusters (Fig. 2I), indicating potential defects in cellular association and migration. A significantly smaller fraction of syndecan-4 $4^{-/}$ satellite cells express MyoD (Fig. 3); those syndecan-4-/cells that do express MyoD at $96 \mathrm{~h}$ very frequently mislocalize the protein to the cytoplasm (Figs. 2I, 3). These data suggest that syndecan- 4 activity is required for normal satellite cell activation and initiation of proliferation, although other factors potentially acting through different signaling pathways may partially compensate for its absence by promoting proliferation. Additionally, syndecan-4 may have an essential role in satellite cell adhesion and/or motility after activation, similar to the effects observed in syndecan-4 ${ }^{-/-}$fibroblasts (Wilcox-Adelman et al. 2002).

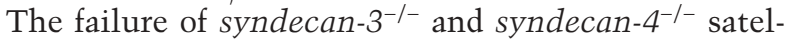
lite cells to express nuclear MyoD appropriately could be expected to result in defects in differentiation. We found that when deprived of association with the host myofiber, more extensive defects in satellite cell function emerge. Although wild-type satellite cell colonies widely express nuclear MyoD and fuse to form elon- gated, multinucleate myotubes (Fig. 4A and insets) that express terminal differentiation markers such as myosin heavy chain (Fig. 4B), both syndecan-null mutants display defects in gene expression and subsequent differentiation. Syndecan- $3^{-/-}$cells proliferate extensively in mass culture but form large, irregular syncytia when attempting to differentiate (Fig. 4C). Furthermore, these cells fail to express MyoD protein either in the nucleus or in the cytoplasm (Fig. 4C and insets) or myosin heavy chain (Fig. 4D), demonstrating a more severe phenotype than that seen on intact myofibers and a general failure to differentiate appropriately in culture. In mass culture, syndecan- $4^{-/}$satellite cells fail at all aspects of the satellite cell program. Compared with wild-type and syndecan- $3^{-/-}$cultures, syndecan- $4^{-/-}$satellite cells are delayed by an additional $48-96 \mathrm{~h}$ before adherent colonies become visible on the culture dish, consistent with the activation and migration defects observed in fiber culture. These cells will continue to express the satellite cell marker c-met, but their myogenic progression is severely impaired: Like syndecan-3-1- cells, they fail to express MyoD (Fig. 4E and insets) or myosin heavy chain (Fig. 4F) and they also fail to fuse with one another (Fig. $4 \mathrm{E}, \mathrm{F})$.

One possible explanation for the observed phenotypes postulates that loss of a specific heparan sulfate may lead to failure to signal through one or more heparan sulfatedependent growth factor receptors. To determine if this mechanism is partially or completely responsible for the defects in satellite cell differentiation in culture, we at-

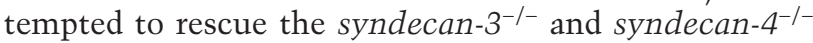
phenotypes by addition of exogenous soluble heparin, which appears to contain all of the sequences found in heparan sulfate. Supplementation with exogenous heparin increased proliferation in mass culture in both wildtype and syndecan- $3^{-/-}$satellite cells but did not alter proliferation in syndecan-4-/- satellite cells (Fig. 4G). A similar result is found when dispersed satellite cells from syndecan-3-1- and syndecan- $4^{-/-}$mice are supplemented with heparin and analyzed for their ability to differentiate. In both genotypes, heparin generally corrects the localization of MyoD to the nucleus (Fig. 4H,I); the ability of syndecan- $3^{-/-}$cells to differentiate appropriately is also significantly improved (Fig. 4H). However, only a marginal improvement in differentiation is seen in syndecan- $4^{-/-}$satellite cells (Fig. 4I) despite the recovery of $\mathrm{MyoD}$ expression and localization. This therefore suggests that either the effects of syndecan-4 loss on satellite cells are primarily due to activities that

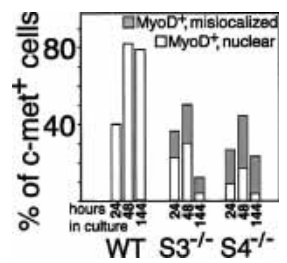

Figure 3. Syndecan-null satellite cells express decreased amounts of MyoD protein and inappropriately localize MyoD protein to the cytoplasm. The fraction of fiber-associated cells as shown in Figure 2 expressing MyoD appropriately in the nucleus or aberrantly in the cytoplasm was determined: Although wild-type cells universally express nuclear MyoD, syndecan-3-/- cells expressing MyoD display cytoplasmic MyoD 40\%-60\% of the time and syndecan-4-1- cells mislocalize MyoD $60 \%-80 \%$ of the time. 


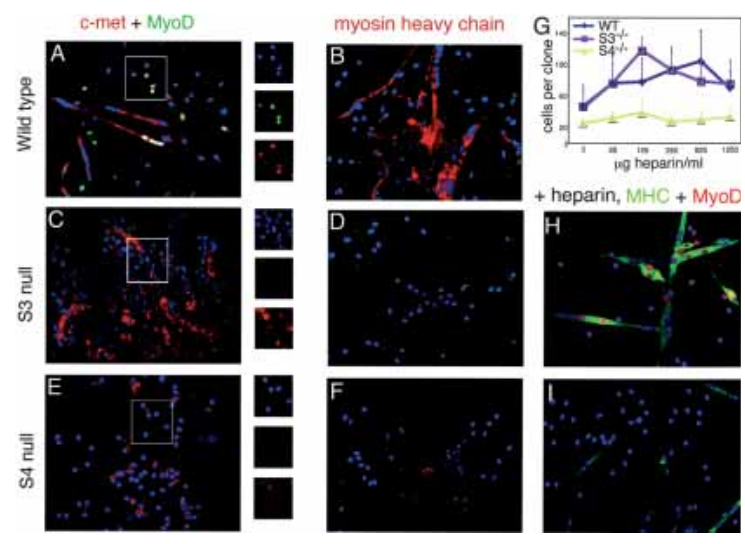

Figure 4. Myogenesis in adherent colonies derived from fiber-associated satellite cells. Satellite cell colonies were stained for c-met (red) and MyoD (green; $A, C, E$ ) or marker myosin heavy chain (red; $B, D, F \mid$; nuclei were visualized with DAPI. Wild-type colonies form multinucleate myotubes, stain positively for $\mathrm{MyoD}(A)$, and express myosin heavy chain, indicating successful differentiation $(B)$. Syndecan-3 $3^{-/}$colonies form large, irregular syncytia, rarely express MyoD $(C)$, and fail to express myosin heavy chain $(D)$. Syndecan- $4^{-/}$ colonies completely fail to either form myotubes or to express MyoD $(E)$ and only very rarely do single cells express myosin heavy chain $(F) .(G)$ Addition of exogenous heparin increased the number of cells per clone in mass cultures of wild-type and syndecan-3-1- satellite cells but not syndecan-4-/- satellite cells; bars represent standard deviations. Heparin also increased differentiation as measured by expression of nuclear MyoD (red) and myosin heavy chain (green) in syndecan-3-/- cells $(525 \mu \mathrm{g} / \mathrm{mL}$ shown; $H)$ and partially rescued the mutant phenotype in syndecan- $4^{-/-}$cells $(525 \mu \mathrm{g} / \mathrm{mL}$ shown; $I)$.

reside in the core protein, or that soluble heparin is insufficient to replace the endogenous syndecan- 4 heparan sulfate.

An appealing hypothesis to explain the differences in phenotype between syndecan-3-/- and syndecan-4- ${ }^{-/}$ satellite cells would be that the individual syndecans are differentially required for binding and signal transduction of different growth factors. The most probable candidates for such signals, based on their known roles in satellite cell biology and the observed results when syndecan-null satellite cells are treated with heparin, are members of the FGF family and hepatocyte growth factor (HGF). Surprisingly, when satellite cells cultured from wild-type, syndecan- $3^{-/-}$, and syndecan $-4^{-/-}$mice are starved, then stimulated with FGF-2 or HGF, either alone or preincubated with soluble heparin, differences in signaling specific to FGF or HGF were not observed. Instead, signaling for both FGF and HGF was similarly affected and dependent on the genotype of the cells. Although wild-type and syndecan- $3^{-1-}$ cells respond to both FGF-2 and HGF by rapid phosphorylation of the ERK1/2 MAP (mitogen-activated protein) kinases, syndecan- $3^{-/-}$cells exhibit a greater than twofold increase in levels of ERK phosphorylation over wild type (Supplementary Fig. 3A). In contrast to wild-type and syndecan$3^{-/-}$cells, syndecan $-4^{-/-}$cells show almost undetectable ERK phosphorylation in response to either FGF or HGF (Supplementary Fig. 3A), indicating a severe impairment of both FGF- and HGF-mediated activation of ERK1/2. When global levels of tyrosine phosphorylation in growing cells of each genotype are examined, syndecan-3-1cells remarkably display an overall increase in phosphorylation (Supplementary Fig. 3B), whereas syndecan$4^{-/-}$cells are similar to wild type. Thus, it appears that syndecan-3-null cells may be missing a key inhibitory signal resulting in a general overstimulation of tyrosine phosphorylation, consistent with the satellite cell and myonuclear hyperplasia. Syndecan- $4^{-/-}$cells appear incompetent for ERK activation through either FGF or HGF receptor tyrosine kinases, yet the global pattern of tyrosine phosphorylation is similar to wild type. This is consistent with the inability of syndecan- $4^{-{ }^{-}}$satellite cells to initially activate and proliferate on myofiber harvest.

These results suggest both the presence of unique defects in syndecan-3-/- and syndecan- $4^{-1-}$ satellite cells, and a requirement for matrix factors not present in ex vivo culture for successful satellite cell function in these cells. Thus, to extend the characterization of the mutant phenotypes and to test this requirement in an in vivo setting, we have analyzed syndecan $-3^{-/-}$and syndecan$4^{-1-}$ tibialis anterior (TA) muscle following barium chloride-induced myonecrosis (Caldwell et al. 1990). Although saline injections failed to induce damage or subsequent regeneration in any genotype (Fig. 5A-C), injection of $1.2 \%$ barium chloride into the TA muscle rapidly induced myonecrosis. At 5 d postinjury in wildtype TA muscle, we consistently observe multiple regenerated myofibers (small-caliber fibers with centrally located nuclei) that appear well organized (Fig. 5D). Syndecan-3 $3^{-1-}$ TA muscle, after $5 \mathrm{~d}$, appears comparable to wild type (Fig. 5E), whereas syndecan-4 ${ }^{-/-}$TA muscle $5 \mathrm{~d}$ postinjury is characterized by poorly organized, irregularly shaped and sized syncytia with highly variable nucleation (Fig. 5F). After $12 \mathrm{~d}$ of regeneration,

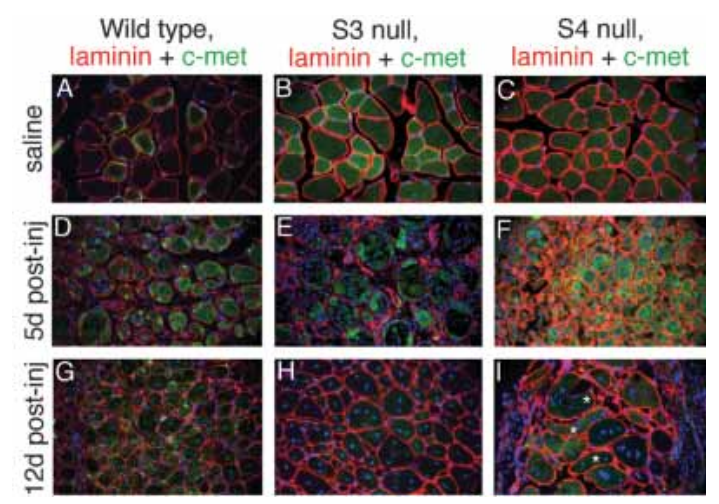

Figure 5. Regeneration was induced by barium chloride injection into the tibialis anterior muscle; control muscles were injected with saline. Sections were stained with antibodies to laminin (red) and the satellite cell marker c-met (green); nuclei were visualized with DAPI. Saline-injected muscles show no increase in regenerating myofibers $(A-C)$, whereas $\mathrm{BaCl}_{2}$ injection rapidly induces myonecrosis and subsequent regeneration as indicated by the presence of centrally nucleated nascent myofibers $(D-F, G-I)$. Wild-type

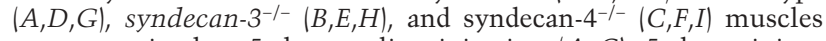
were examined at $5 \mathrm{~d}$ postsaline injection $(A-C), 5 \mathrm{~d}$ postinjury $(D-F)$, and $12 \mathrm{~d}$ postinjury $(G-I)$. $(F)$ Wild-type and syndecan-3muscles have reconstituted myofiber patterning by $5 \mathrm{~d}$ postinjury; however, syndecan-4-1- muscles have neither well-defined nascent myofibers nor well-patterned muscle. At $12 \mathrm{~d}$ postinjury, wild-type $(G)$ and syndecan-3-1- $(H)$ muscles have regenerated with similar efficiencies, although syndecan $-3^{-/-}$regenerates display hyperplasia similar to that seen in uninjured muscle. (I) In contrast, syndecan$4^{-/-}$regenerates after $12 \mathrm{~d}$ have not replaced the damaged myofibers or reformed fibers that do appear into appropriately patterned muscle; asterisks indicate nascent myofibers that are not aligned with the axis of the regenerating muscle. 
wild-type TA muscle has continued to regenerate, producing closely packed nascent myofibers (Fig. 5G), as has syndecan-3-1- TA muscle (Fig. $5 \mathrm{H})$; however, syndecan $-3^{-1-}$-regenerated TA muscle is also characterized by extensive myonuclear and satellite cell hyperplasia that appears similar to all limb muscle formed during development (data not shown). In marked contrast, after $12 \mathrm{~d}$ of regeneration, syndecan $-4^{-1-}$ TA muscle has not regenerated and is composed primarily of disorganized, nonfunctional myofibers and nonmuscle scar tissue (Fig. 5I). Note that some fibers that appear are so disorganized that they do not align with the axis of the former myofibers (asterisks, Fig. 5I). Unlike wild-type and syndecan$3^{-/-}$mice, which will begin to bear weight on the injured leg within 2-3 d after injury, injured syndecan-4-/- animals continue to favor the injured leg for at least $4 \mathrm{wk}$ postinjury (data not shown). Interestingly, the expression patterns of syndecan- 3 and syndecan- 4 diverge during regeneration in wild-type muscle, the first such instance we have observed. During acute regeneration, syndecan-4 immunoreactivity is increased in the activated satellite cells, whereas syndecan-3 immunoreactivity becomes preferentially localized to the nascent myofibers (data not shown). Following regeneration, the normal pattern of coexpression on quiescent satellite cells is reestablished.

These results demonstrate unique requirements for each syndecan in skeletal muscle. Syndecan-3-1- muscle is characterized by mild hypotonia, fibrosis, and chronic regeneration, suggesting defects in differentiated muscle that may describe a novel form of muscular dystrophy. Another striking phenotype is the increase in both myofiber nuclei and satellite cell nuclei over wild type, which is maintained even after an acute round of induced myonecrosis and regeneration. Although indirect, these data may be interpreted to suggest that precursors for differentiated myonuclei and satellite cells both experience a similar syndecan-3-dependent attenuation of proliferation. The lineal origin of satellite cells is not known, as they have alternatively been proposed to be derived from developing myoblasts, the adult myoblast population, bone marrow-derived stem cells, and vesselassociated mesangioblasts (for review, see Seale and Rudnicki 2000). Because the satellite cells and myonuclei exhibit a similar hyperplasia, our data would support the hypothesis that differentiated muscle precursors and satellite cell precursors share common regulatory mechanisms and may either share a common progenitor or may occupy a common niche during development.

The disparities observed between the myogenic capacity of syndecan-3-1- satellite cells in vivo (as demonstrated by the high percentage of regenerating myofibers in uninjured muscle and after induced regeneration), in fiber culture, and in adherent culture suggest a requirement for additional matrix factors to achieve appropriate myogenesis in syndecan- $3^{-/-}$satellite cells. This is likely to include heparan sulfate provided by other HSPGs such as perlecan, which is expressed at higher levels in the matrix of syndecan-3 $3^{-1-}$ muscle (data not shown). This conclusion is consistent with the rescue of differentiated morphology and gene expression in syndecan-3-/- satellite cells by addition of exogenous heparin. In contrast, whereas syndecan- $4^{-/-}$muscle tissue is significantly less affected than syndecan $-3^{-/-}$muscle, syndecan-4-/- satellite cells are much more severely impaired than syndecan- $3^{-/-}$cells, as they both fail to behave normally in culture and fail to regenerate functional muscle in vivo. Rescue of the syndecan-4-1- cells in mass culture by exogenous heparin is also less efficient than in syndecan$3^{-/-}$satellite cells.

Our data demonstrate that the surrounding environment is critical for satellite cell proliferation and differentiation. These experiments have demonstrated that syndecan-3 and syndecan-4 play distinct roles in muscle development as well as in satellite cell physiology and that the molecular and cellular consequences of deleting either syndecan are both complex and context dependent. We have initiated mRNA analysis of quiescent and activated satellite cells directly harvested from intact or injured hindlimb muscle of wild-type and syndecan-null mice. Preliminary analysis of these data suggests that syndecan- $3^{-1-}$ and syndecan- $4^{-/-}$satellite cells differ significantly from each other as well as from wild-type satellite cells. Syndecan-3 $3^{-/}$cells most resemble wild-type cells (D. Cornelison, T. Cheung, X. Liu, and B. Olwin, in prep.), as might be expected from their cellular phenotype. Further analysis of the gene expression profiles between the syndecan-3-null and syndecan-4-null satellite cells, coupled with biochemical characterization of affected signaling pathways, is expected to identify molecular differences between these genotypes critical for satellite cell function.

\section{Materials and methods}

Mice

All genotypes of mice used were housed in the same pathogen-free facility at the University of Colorado; all mice used were females between 80 and $160 \mathrm{~d}$ of age. Wild-type mice were C57Bl/6xDBA2 (B6D2F1; Jackson Labs); syndecan- $3^{-/-}$mice carry the homozygous deletion of syndecan-3 on a C57Bl/6 background (Kaksonen et al. 2002), and syndecan- $4^{-/-}$mice carry the homozygous deletion of syndecan- 4 on a $\mathrm{C} 57 \mathrm{Bl} / 6$ background (Echtermeyer et al. 2001).

Sectioning and immunostaining of fixed frozen sections

For Oil Red O staining, sections were equilibrated into propylene glycol, stained with $0.7 \%$ Oil Red O in propylene glycol, destained in $85 \%$ propylene glycol, and extensively washed with water. For fluorescent staining, sections were blocked in 10\% BlokHen (Aves) if chicken antisyndecan- 4 was to be used, and/or $10 \%$ normal goat serum in phosphatebuffered saline. Primary antibodies used were rabbit anti-laminin (Sigma) at 1:200, mouse anti-neurofilament (Sigma) at 1:100, rabbit anti-syndecan-3 at 1:100, chicken anti-syndecan-4 at 1:1500, rabbit anti-c-met (Santa Cruz) at 1:100, mouse anti-MyoD (Novocastra) at 1:10, and mouse anti-myosin heavy chain (MF20) neat. Secondary antibodies conjugated to Alexa 488 or Alexa 594 (Molecular Probes) were used at 1:500.

Myofiber preparation and staining

Single myofibers were isolated, cultured, and stained as described previously (Cornelison et al. 2001), except that the culture medium used was Ham's F-12 supplemented with $15 \%$ horse serum and 0.5 nM FGF-2. Fibers at designated times after harvest were fixed and stained with antibodies as described earlier.

Adherent culture

Single myofibers $24 \mathrm{~h}$ after isolation were plated in growth medium in tissue culture dishes containing gelatin-coated glass coverslips and left undisturbed for $6 \mathrm{~d}$. Soluble heparin (Sigma) was added as indicated from a $50 \mathrm{mg} / \mathrm{mL}$ stock. The colonies formed by emigrating satellite cells on the coverslips were fixed and stained as described earlier.

In vivo regeneration

Mice were anesthetized with $5 \%$ Avertin $(175 \mu \mathrm{L} /$ mouse $)$. Left TA muscles were injected with $50 \mu \mathrm{L}$ sterile saline and the contralateral TA 


\section{Cornelison et al.}

muscles were injected with $50 \mu \mathrm{L} 1.2 \% \mathrm{BaCl}_{2}$ in sterile saline. Mice were allowed to recover for 5 or $12 \mathrm{~d}$, then injected muscles were harvested for sectioning and staining as described earlier.

\section{Acknowledgments}

This work was supported by a grant from the MDA to D.D.W.C., grants from the MDA and NIH (AR39467) to B.B.O., NIH grants HD21881 and GM48850 to A.C.R., and AHA (0250523N) and NIH (HD 37490) grants to P.F.G.

\section{References}

Allen, R.E., Sheehan, S.M., Taylor, R.G., Kendall, T.L., and Rice, G.M. 1995. Hepatocyte growth factor activates quiescent skeletal muscle satellite cells in vitro. J. Cell. Physiol. 165: 307-312.

Caldwell, C.J., Mattey, D.L., and Weller, R.O. 1990. Role of the basement membrane in the regeneration of skeletal muscle. Neuropathol. Appl. Neurobiol. 16: 225-238.

Carey, D.J. 1997. Syndecans: Multifunctional cell-surface co-receptors. Biochem. J. 327: 1-16.

Cornelison, D.D.W. and Wold, B.J. 1997. Single-cell analysis of regulatory gene expression in quiescent and activated mouse skeletal muscle satellite cells. Dev. Biol. 191: 270-283.

Cornelison, D.D., Olwin, B.B., Rudnicki, M.A., and Wold, B.J. 2000. $\mathrm{MyoD}^{-/-}$satellite cells in single-fiber culture are differentiation defective and MRF4 deficient. Dev. Biol. 224: 122-137.

Cornelison, D.D.W., Filla, M.S., Stanley, H.M., Rapraeger, A.C., and O1win, B.B. 2001. Syndecan-3 and syndecan-4 specifically mark skeletal muscle satellite cells and are implicated in satellite cell maintenance and muscle regeneration. Dev. Biol. 239: 79-94.

Echtermeyer, F., Streit, M., Wilcox-Adelman, S., Saoncella, S., Denhez, F., Detmar, M., and Goetinck, P. 2001. Delayed wound repair and impaired angiogenesis in mice lacking syndecan-4. J. Clin. Invest. 107: R9-R14.

Hawke, T.J. and Garry, D.J. 2001. Myogenic satellite cells: Physiology to molecular biology. J. Appl. Physiol. 91: 534-551.

Kaksonen, M., Pavlov, I., Voikar, V., Lauri, S.E., Hienola, A., Riekki, R., Lakso, M., Taira, T., and Rauvala, H. 2002. Syndecan-3-deficient mice exhibit enhanced LTP and impaired hippocampus-dependent memory. Mol. Cell. Neurosci. 21: 158-172.

Larrain, J., Cizmeci-Smith, G., Troncoso, V., Stahl, R.C., Carey, D.J., and Brandan, E. 1997. Syndecan-1 expression is down-regulated during myoblast terminal differentiation. Modulation by growth factors and retinoic acid. J. Biol. Chem. 272: 18418-18424.

Olguin, H. and Brandan, E. 2001. Expression and localization of proteoglycans during limb myogenic activation. Dev. Dyn. 221: 106-115.

Ornitz, D.M. 2000. FGFs, heparan sulfate and FGFRs: Complex interactions essential for development. Bioessays 22: 108-112.

Rapraeger, A.C. 2000. Syndecan-regulated receptor signaling. J. Cell Biol. 149: 995-998.

Reizes, O., Lincecum, J., Wang, Z., Goldberger, O., Huang, L., Kaksonen, M., Ahima, R., Hinkes, M.T., Barsh, G.S., Rauvala, H., et al. 2001. Transgenic expression of syndecan-1 uncovers a physiological control of feeding behavior by syndecan-3. Cell 106: 105-116.

Schultz, E. and McCormick, K.M. 1994. Skeletal muscle satellite cells. Rev. Physiol. Biochem. Pharmacol. 123: 213-257.

Seale, P. and Rudnicki, M.A. 2000. A new look at the origin, function, and "stem-cell" status of muscle satellite cells. Dev. Biol. 218: 115-124.

Tatsumi, R., Anderson, J.E., Nevoret, C.J., Halevy, O., and Allen, R.E. 1998. HGF/SF is present in normal adult skeletal muscle and is capable of activating satellite cells. Dev. Biol. 194: 114-128.

Watchko, J.F., O'Day, T.L., and Hoffman, E.P. 2002. Functional characteristics of dystrophic skeletal muscle: Insights from animal models. I. Appl. Physiol. 93: 407-417.

Wilcox-Adelman, S.A., Denhez, F., and Goetinck, P.F. 2002. Syndecan-4 modulates focal adhesion kinase phosphorylation. J. Biol. Chem. 277: 32970-32977.

Zimmermann, P. and David, G. 1999. The syndecans, tuners of transmembrane signaling. FASEB J. 13: S91-S100. 


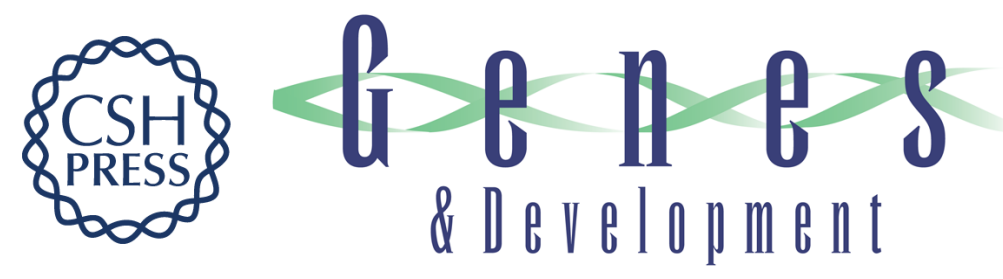

\section{Essential and separable roles for Syndecan-3 and Syndecan-4 in skeletal muscle development and regeneration}

D.D.W. Cornelison, Sarah A. Wilcox-Adelman, Paul F. Goetinck, et al.

Genes Dev. 2004, 18:

Access the most recent version at doi:10.1101/gad.1214204

Supplemental
Material http://genesdev.cshlp.org/content/suppl/2004/09/02/18.18.2231.DC1

References This article cites 18 articles, 3 of which can be accessed free at:

http://genesdev.cshlp.org/content/18/18/2231.full.html\#ref-list-1

License

Email Alerting Receive free email alerts when new articles cite this article - sign up in the box at the top

Service right corner of the article or click here.

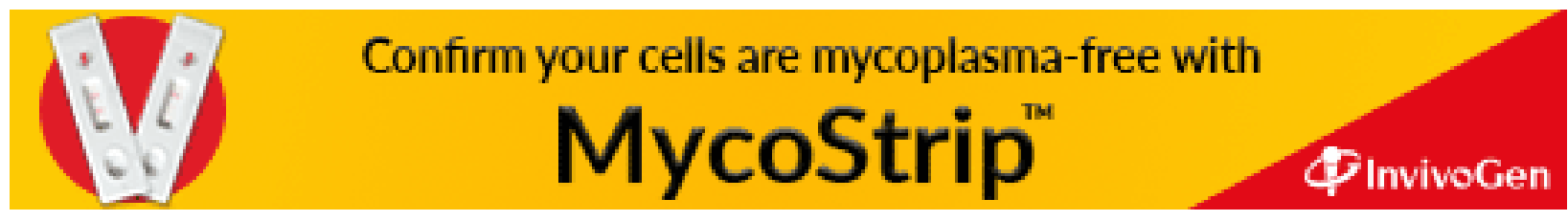

